\title{
Saccadic Dysmetria and Adaptation after Lesions of the Cerebellar Cortex
}

\author{
Shabtai Barash, ${ }^{1}$ Armenuhi Melikyan, ${ }^{1}$ Alexey Sivakov, ${ }^{1}$ Mingsha Zhang, ${ }^{1}$ Mitchell Glickstein, ${ }^{2}$ and \\ Peter Thier ${ }^{3}$ \\ ${ }^{1}$ Department of Neurobiology, Weizmann Institute of Science, Rehovot 76100, Israel, ${ }^{2}$ Department of Anatomy, University \\ College London, London WC1E 6BT, United Kingdom, and ${ }^{3}$ Sektion für Visuelle Sensomotorik, Neurologische \\ Universitätsklinik Tübingen, 72076 Tübingen, Germany
}

\begin{abstract}
We studied the effects of small lesions of the oculomotor vermis of the cerebellar cortex on the ability of monkeys to execute and adapt saccadic eye movements. For saccades in one horizontal direction, the lesions led to an initial gross hypometria and a permanent abolition of the capacity for rapid adaptation. Mean saccade amplitude recovered from the initial hypometria, although variability remained high. A series of hundreds of repetitive saccades in the same direction resulted in gradual decrement of amplitude. Saccades in other directions were less strongly affected by the lesions. We suggest the
\end{abstract}

following. (1) The cerebellar cortex is constantly recalibrating the saccadic system, thus compensating for rapid biomechanical changes such as might be caused by muscle fatigue. (2) A mechanism capable of slow recovery from dysmetria is revealed despite the permanent absence of rapid adaptation.

Key words: saccades; saccadic eye movement; saccadic adaptation; fatigue; cerebellum; vermis; dysmetria; lesion; eye movement; recovery; recovery from brain damage; motor learning; cerebellar cortex
Accurate saccadic eye movements are critical for directing the fovea to sites of interest in a given visual scene. The posterior vermis of the cerebellar cortex and its major output, the fastigial nucleus, are related to the control of saccadic accuracy and to saccadic adaptation (for review, see Leigh and Zee, 1990; Botzel et al., 1993; Buettner and Fuhry, 1995). Extensive studies by Noda and colleagues delineated a confined saccadic area in the posterior vermis (Fujikado and Noda, 1987; Noda and Fujikado, 1987a,b; Yamada and Noda, 1987; Sato and Noda, 1992; Ohtsuka and Noda, 1995).

If the fastigial nucleus is ablated with the cortex of the vermis (Ritchie, 1976; Optican and Robinson, 1980) or without it (Goldberg et al., 1993), saccades become permanently grossly hypermetric, and the capacity for adaptation is lost. Are these findings but indirect effects of blocking out the posterior vermis, Noda's area in particular? This question is of obvious interest for understanding the role of the cerebellar cortex and nuclei in motor learning (Glickstein, 1992; Raymond et al., 1996; Thompson et al., 1997; Ito, 1998). To date, the literature contains only a single report of a monkey with a bilateral lesion of Noda's area, which was tested for an extended postoperative period [Takagi et al. (1998), their Monkey 1]. In other studies, the animals were tested after a brief postoperative period (Aschoff and Cohen, 1971; Sato and Noda, 1992), or the lesion included the fastigial nucleus or cerebellar cortex beyond the posterior vermis (Ritchie, 1976; Optican and Robinson, 1980). Two of Takagi et al.'s (1998) monkeys had predominantly unilateral lesions, and in these animals, the deficits were not the same as those found in their

Received May 4, 1999; revised Aug. 25, 1999; accepted Aug. 25, 1999.

This work was supported by a grant from the Germany-Israel Foundation for Scientific Research and Development.

Correspondence should be addressed to Dr. Shabtai Barash, Department of Neurobiology, Weizmann Institute of Science, Rehovot 76100, Israel. E-mail: shabtai.barash@weizmann.ac.il.

Copyright (C) 1999 Society for Neuroscience 0270-6474/99/1910931-09\$05.00/0
Monkey 1. Residual function after a partial lesion might generally be mediated by the remaining part of Noda's area. For unilateral lesions, this is particularly relevant because the two fastigial nuclei, and hence the two sides of the vermis, maintain a delicate dynamic balance of push-pull interactions (Robinson et al., 1993). Even if complete lesions of the saccadic posterior vermis were to cause saccadic hypometria, unilateral lesions might still cause hypermetria because of the imbalance they create (Buettner and Fuhry, 1995).

Two types of saccadic adaptation were traditionally thought to exist. Slow adaptation (days) is revealed in the recovery from extraocular muscle paresis (Kommerell et al., 1976; Optican and Robinson, 1980). Rapid adaptation (within a session) can be achieved by shifting the target while a saccade is under way (McLaughlin, 1967; Fitzgibbon et al., 1986; Albano and King, 1989; Straube et al., 1997). The two procedures might invoke the same physiological process (Scudder et al., 1998). We will propose that rapid adaptation is not a mere laboratory trick devoid of physiological function. Rather, one important function of rapid adaptation is to overcome changes caused by fatigue. Furthermore, a very slow process of recovery from dysmetria is anatomically separable from rapid adaptation because it survives the cerebellar cortical lesion, which abolishes rapid adaptation.

\section{MATERIALS AND METHODS}

Our general procedures were recently described in detail (Barash et al., 1998). The experiments were conducted in a light-tight, sound attenuated, electrically isolated room. The visual background was uniform dim gray $\left(1 \mathrm{~cd} / \mathrm{m}^{2}\right)$. A computer display system was projected on a large back-projection screen positioned $86 \mathrm{~cm}$ in front of the animals. The vertical refresh rate was $77 \mathrm{~Hz}$; hence, the temporal resolution was 13 msec. The monkey sat in a primate chair with its head fixed. Eye position was measured by a scleral search coil system and sampled at $500 \mathrm{~Hz}$ with 16 bit resolution. Saccade onset was determined on-line by monitoring the change in eye position. Because of this procedure and the delays inherent in the raster system, the target shift in adaptation trials may have 
occurred up to $20 \mathrm{msec}$ after saccade onset, as determined post hoc. Nevertheless, systematic saccadic adaptation was obvious before the lesions.

Each monkey was tested postoperatively in two periods. The "early postlesion" period spanned the first two postlesion weeks (but saccadic dysmetria studies were limited to the first week). The "late postlesion" period spanned days 283-409 in monkey 1 and days 54-103 in monkey 2. Additional visuomotor tasks were tested that are not reported here.

Each of the two male Macaca fascicularis monkeys went through two surgical procedures. First, they were implanted with a scleral search coil (Judge et al., 1980) and a head post. The cerebellar lesion was made later, after the monkeys were trained to adequately perform their tasks. Surgeries were conducted in sterile aseptic conditions under general anesthesia. The electrocardiogram was monitored, and body temperature was fixed at $37^{\circ} \mathrm{C}$. Sterile orthopedic bone cement and bone screws were used. The initial implantation procedure is standard (Barash et al., 1998). To make the lesion, the neck muscles were cut and separated at the midline. A small craniectomy was made below the occipital protuberance. After the dura overlying the cerebellum was exposed, mannitol was slowly administered. A dural flap was made exposing the caudal vermis and neighboring regions of the hemispheres. Figure 12 of Noda and Fujikado (1987a) was particularly helpful for identifying Noda's area according to its relationship to the sulcal pattern of the cerebellum, in particular to the junction of the simple lobule and crus II. (Fortunately, in both of our monkeys, the landscape was similar to Noda and Fujikdo's Figure 12A,B and not $C, D)$. A lesion was made with a fine-gauge sucker. When hemostasis was complete, the dura, muscles, and skin were sutured in anatomical layers.

Recovery from the cerebellar lesion was very rapid. Within hours from completion of surgery, the monkeys behaved normally, and there were no overt neurological signs. For 1 week after surgeries, the monkeys were transferred to the chair to care for the wounds and for oral administration of analgesics and antibiotics. Beginning on the second day after the lesion surgery, while the monkeys were taken out of the cage for their medical care, the chairs were positioned in the experimental setup and the monkeys were given opportunity to perform saccade trials. Although the monkeys were not deprived of water or food, they were invariably willing to perform the task. These rather brief sessions allowed us to map saccadic dysmetrias before any recovery took place. Adaptation was not studied in the early postlesion period.

At the completion of the experiment ( 1 year and 3 months after the lesion surgery, respectively) the monkeys were deeply anesthetized by a lethal dose of Nembutal and perfused with saline, followed by fixative. The cerebellum was sectioned parasagittally, and the brainstem was sectioned horizontally in $60 \mu \mathrm{m}$ sections and Nissl stained. Experimental procedures follow the United States National Institutes of Health Guidelines and Israeli law and were approved by the Animal Care and Use Committee of the Weizmann Institute.

\section{RESULTS}

The lesion-induced deficits in saccadic accuracy and adaptation that we observed were substantial primarily in one or both horizontal directions, whereas the vertical and other horizontal directions were less affected. In the first part of Results, we focus on the deficits in the directions strongly affected by the lesions.

\section{Saccadic adaptation in the intact monkey}

Saccadic adaptation, as observed before the cerebellar lesions, is illustrated in Figure 1. We used the procedure introduced by McLaughlin (1967). The same initial fixation and target positions are used in all trials $\left(15^{\circ}\right.$ to the right in the block illustrated in Fig. 1). Trials are arranged in three successive groups that are run one after another, without pauses. The first, baseline group consists of control trials (Fig. 1A). The target jump is followed by a saccade. Control saccades were similar to each other, and there was minimal variability in saccade parameters, such as saccade size, maximal velocity, etc.

Adaptation trials began in the same way as the control trials, but shortly after the saccade onset and while the saccadic movement was under way, the target was displaced from $15^{\circ}$ to $20^{\circ}$ eccentricity. Figure $1 B$ shows the first adaptation trial. The sac-
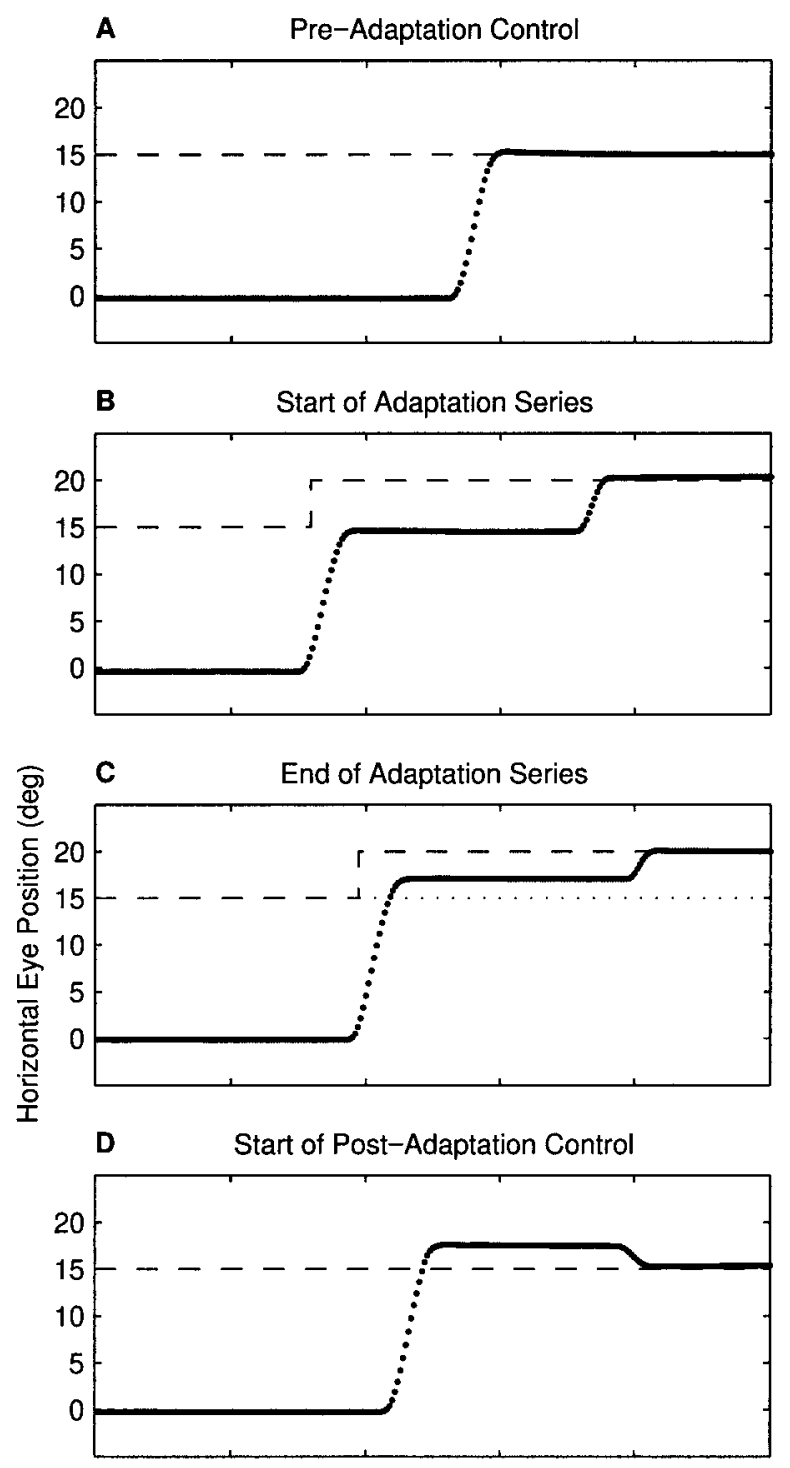

E End of Post-Adaptation Control

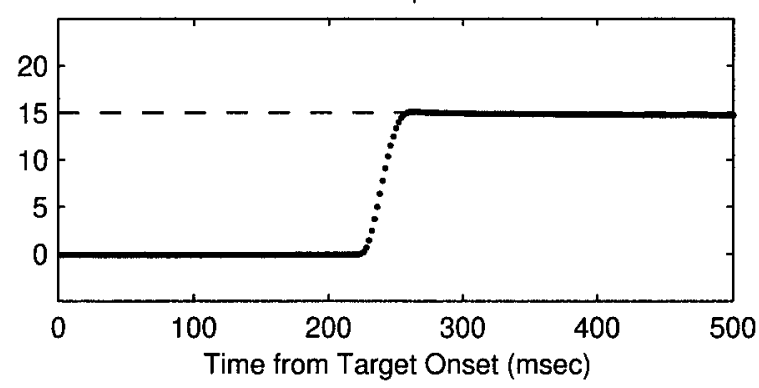

Figure 1. Examples of trials illustrating saccadic adaptation and extinction. Each panel shows the horizontal eye position and target position during trials from a block designed to study saccadic adaptation. Rightward saccades. A full record of saccade sizes in this block is shown in Figure $2 B$.

cade ends very close to the $15^{\circ}$ location, where the target had been before the saccade, so that the eye position is $5^{\circ}$ short of the target. McLaughlin (1967) compared the subject's situation at the end of the saccade, finding itself short of the target, with the similar situation that would occur if the monkey's extraocular 
Monkey 1

Monkey 2

\section{Before Lesion}

A

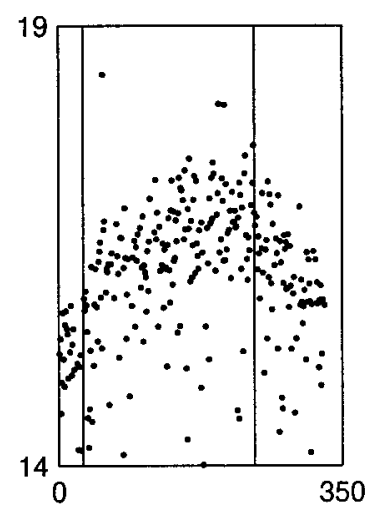

B

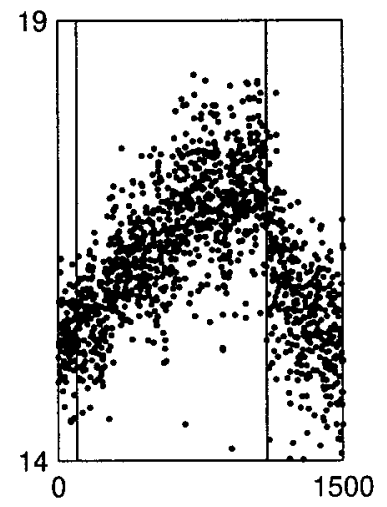

After Recovery from Dysmetria
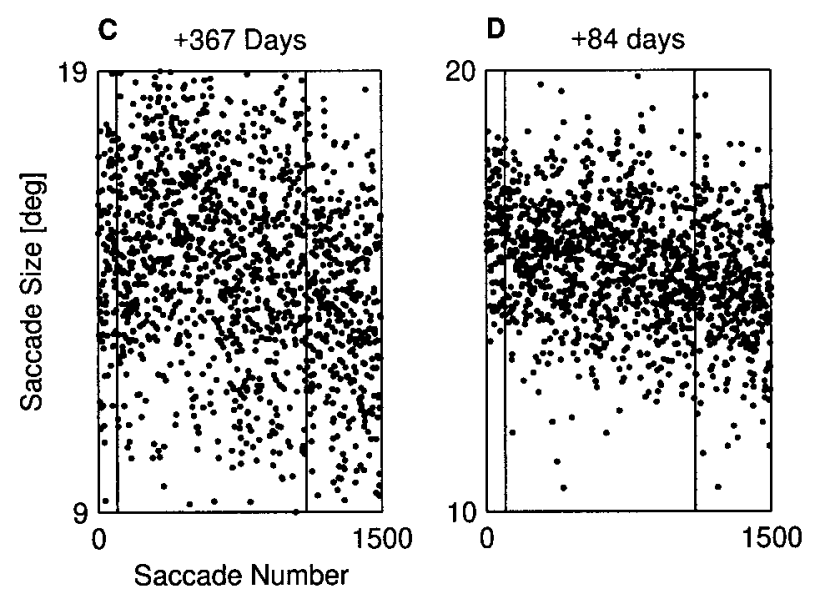

Figure 2. Saccadic adaptation is abolished by the cerebellar lesion. Panels show full records of saccade sizes for block of trials recorded at the days indicated above the panels. All movements are to the right. The two vertical lines in each panel indicate the beginning and end of adaptation trials. The scale of $A$ and $B$ is stretched twice relative to $C$ and $D$.

muscles were suddenly weakened. The monkey responds with a second saccade to fixate the new target position.

The pattern of visual stimulation in the adaptation trial illustrated in Figure $1 C$ is identical to that of the trial of Figure $1 B$; the only difference is that, whereas Figure $1 B$ shows the first adaptation trial in the displayed block, Figure $1 C$ shows the 1000 th consecutive adaptation trial. The monkey's responses are now very different. The eye overshoots the presaccadic position of the target, reflecting the monkey's recent experience with the previous 1000 adaptation trials. Thus, Figure $1 C$ replicates McLaughlin's observation of a change in saccadic gain induced by the repeated intersaccadic target jump. Figure $2 B$ shows the full record of the saccade sizes from the block illustrated in Figure 1. Saccade size increases very gradually during the adaptation trials.

Figure $1 D$ shows a control saccade trial in which the target remains at its presaccadic position after the series of 1000 adaptation trials. Although the initial target position is the same as in the control trial of Figure $1 A$, the saccade now overshoots the target. The third group of trials was designed to study the extinc- tion of adaptation. Figure $1 D$ shows the first of these. Repetition of control trials leads to extinction of the adaptation; the monkey's saccades are recalibrated to their normal size (Fig. 1E). Figure $2 B$ shows that extinction also occurs gradually, but more rapidly than acquisition of adaptation.

\section{Abolition of adaptation}

The vermian lesion permanently abolished rapid adaptation of the amplitudes of rightward saccades (Fig. 2). Figure 2, $C$ and $D$, illustrates postlesion studies of saccadic adaptation. Although the procedures were identical to these of the prelesion studies illustrated in Figure 2, $A$ and $B$, the results differ greatly. Adaptation is abolished. Despite 1000 adaptation trials, there is no increase in saccade size, nor is there any sign of extinction effects caused by the switch back from adaptation to control trials. These changes persist to the late postlesion phase, $\sim 3$ months and 1 year after the lesion. Thus, the abolition of adaptation is permanent.

Two additional effects of vermian lesions can be discerned in Figure 2. First, saccade end points are more variable after the lesion (note the difference in scale between Fig. $2 A, B$ and $C, D$ ). Second, during these long series of adaptation trials, saccade size is continuously reduced. We will return to these effects below.

\section{Saccadic hypometria}

Figure 3 illustrates eye position records of ten $15^{\circ}$ saccades in the most severely dysmetric direction for each monkey. Prelesion movements are swift and precise (Fig. $3 A, D$ ). Immediately after the lesion (day +3$)$, fixation is less precise and saccades are grossly dysmetric (Fig. 3B,E). After three months (monkey 2) and 1 year (monkey 1) recovery of average saccade amplitude is nearly complete, although now more variable than preoperatively (Fig. $3 C, F$ ). The gain in these early postlesion trials is, respectively, 79 and $65 \%$. Early postlesion saccades are highly significantly different from both prelesion and late postlesion saccades ( $p<10^{-6}$ in both monkeys). In contrast, the mean size of late postlesion saccades is not significantly different from prelesion ( $p=0.55$ and 0.24 , respectively; all values by $t$ test).

Figure 4 documents both the hypometria and recovery more thoroughly. The figure shows histograms of saccade size for all the control saccades in the affected directions recorded in this study (464 and 1443 saccades, respectively, in the two monkeys). The data of the late postlesion recovery was collected over 39 experimental days (22 d in monkey 1 and $17 \mathrm{~d}$ for monkey 2). There were no systematic differences among days. Early postlesion saccades are highly significantly different from both prelesion and late postlesion saccades $\left(p<10^{-15}\right)$. In contrast, the mean size of the late postlesion saccades is virtually the same as that of the prelesion saccades in both monkeys. The large sample sizes that we used argue that the lack of a significant difference between the means is evidence that the mean sizes of prelesion and late postlesion saccades are indeed nearly perfectly equal.

Despite their entirely recovered mean amplitude, late postlesion saccades remain abnormal. They are also slower, the maximal saccadic velocity being reduced from $622 \pm 29 \%$ sec in the prelesion period to $553 \pm 41 \% \mathrm{sec}$ in the late postlesion period in monkey 1 and from $641 \pm 47 \%$ sec to $620 \pm 52 \%$ sec in monkey 2 . Both differences are significant with $p<10^{-6}$ ( $t$ test). Note that we can directly compare dynamic parameters of dysmetric versus intact saccades only because of the almost perfect recovery of the mean saccade size. Generally such comparisons are confounded by the strong dependence of dynamics on saccade size. 


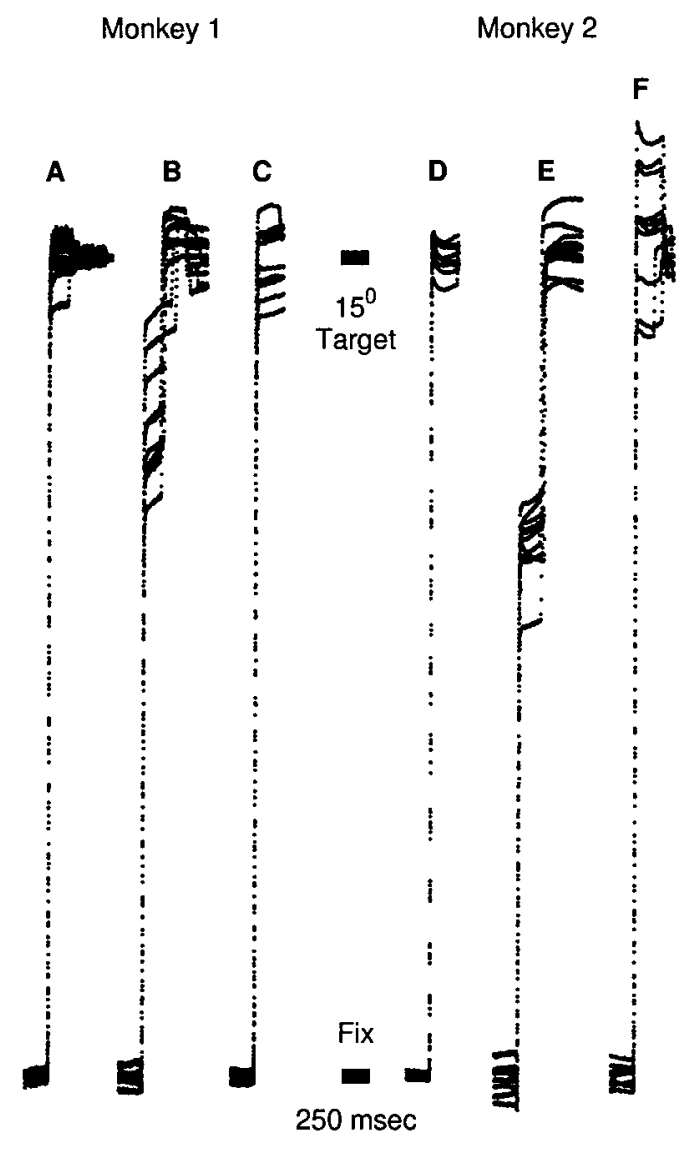

Figure 3. Lesions of the oculomotor vermis induce gross dysmetria, but with time the dysmetria recovers. Saccades from prelesion period $(A, D)$, early postlesion (day +3$)(B, E)$, and late postlesion (3 months, 1 year) $(C$, $F)$. Monkey $1(A-C)$, leftward saccades; monkey $2(D-F)$, rightwards saccades. Ten saccades per panel. In trials early after the lesion, two and sometimes three saccades are needed to fixate the target $(B, E)$. Whereas the mean saccade size recovers $(C, F)$, the variability in both fixation and saccade size persists $(B, C, E, F)$.

\section{Postlesion continuous reduction of saccade size: fatigue?}

A second, unexpected effect of the lesions was a continuous reduction in saccade amplitude over long blocks of identical saccades (Fig. 2). This slow reduction is continuous throughout the block, regardless of whether trials are of the adaptation or extinction stages. The continuous reduction was somewhat more pronounced in monkey 2 than in monkey 1 , although it was clearly present in both. The continuous reduction in amplitude might result from the monkey's increasing fatigue that is presumably caused by repeating the same movement over and over. The cerebellar area ablated in our monkeys may thus normally serve to counteract fatigue-related saccadic inaccuracy.

We tested whether the continuous reduction might be caused by the unnatural pattern of visual stimulation that occurs in the adaptation paradigm. To evaluate whether the continuous reduction is indeed specific to blocks of adaptation trials, we tested monkey 2 in a control experiment in which the adaptation trials were replaced by trials with a single $20^{\circ}$ saccade. Before and after the $20^{\circ}$ saccades, there were groups of $15^{\circ}$ saccade trials, just as in the adaptation studies. Saccade size is continuously reduced in these control conditions as well (Fig. 5A). The continuous reduction is particularly apparent when the $15^{\circ}$ saccades at the begin-
Monkey $1 \quad$ Monkey 2
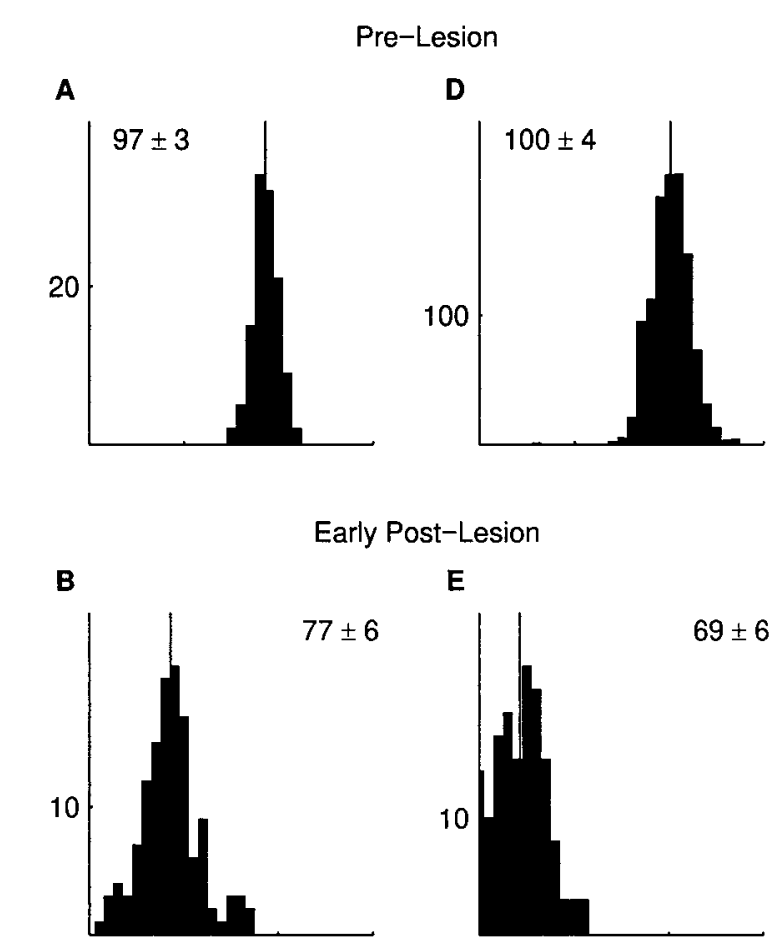

E

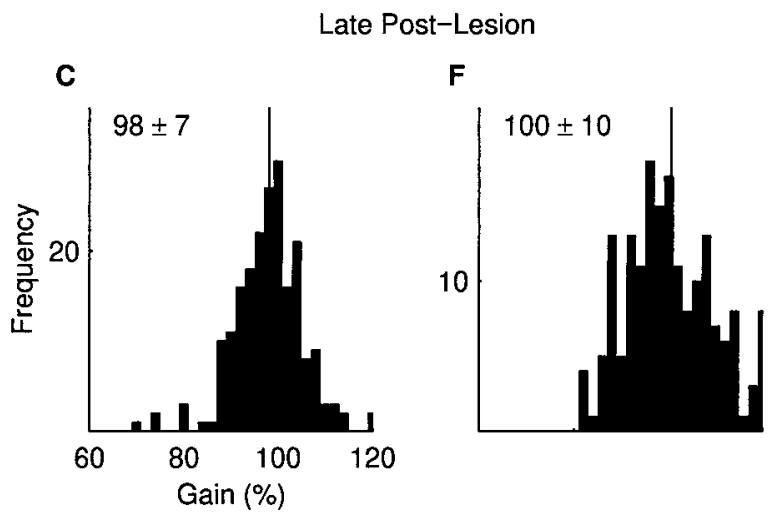

Figure 4. Histograms of saccade size documentating postlesion hypometria and recovery. $A-C$, Monkey $1 ; D-F$, monkey 2 . $A$ and $D$ represent prelesion period; $B$ and $E$ show early postlesion period; $C$ and $F$ show late postlesion period. Saccades in same directions as Figure 1. All appropriate control saccades recorded in this study are included. Thick vertical lines represent means of distributions. Values on each panel show mean \pm $\mathrm{SD}$ of gain (in percents).

ning and the end of the block are compared directly. Figure $5 B$ is a histogram of the first $10015^{\circ}$ saccades in the block, and Figure $5 C$ is a histogram of the last 100 trials. Mean saccade size drops from 15.2 to $14.1^{\circ}$. The difference is highly significant $\left(p<10^{-15}\right.$; $t$ test). For comparison, we tested a third, intact monkey in the same control paradigm. The results are presented in Figure $5 D-F$. The intact monkey made more precise and less variable saccades, without reduction of saccade size. The distributions of the first and last 100 saccades in the block (Fig. $5 E, F$ ) are similar, and the means are not statistically different $(p=0.3)$. Therefore, the continuous reduction is not a by-product of the adaptation 

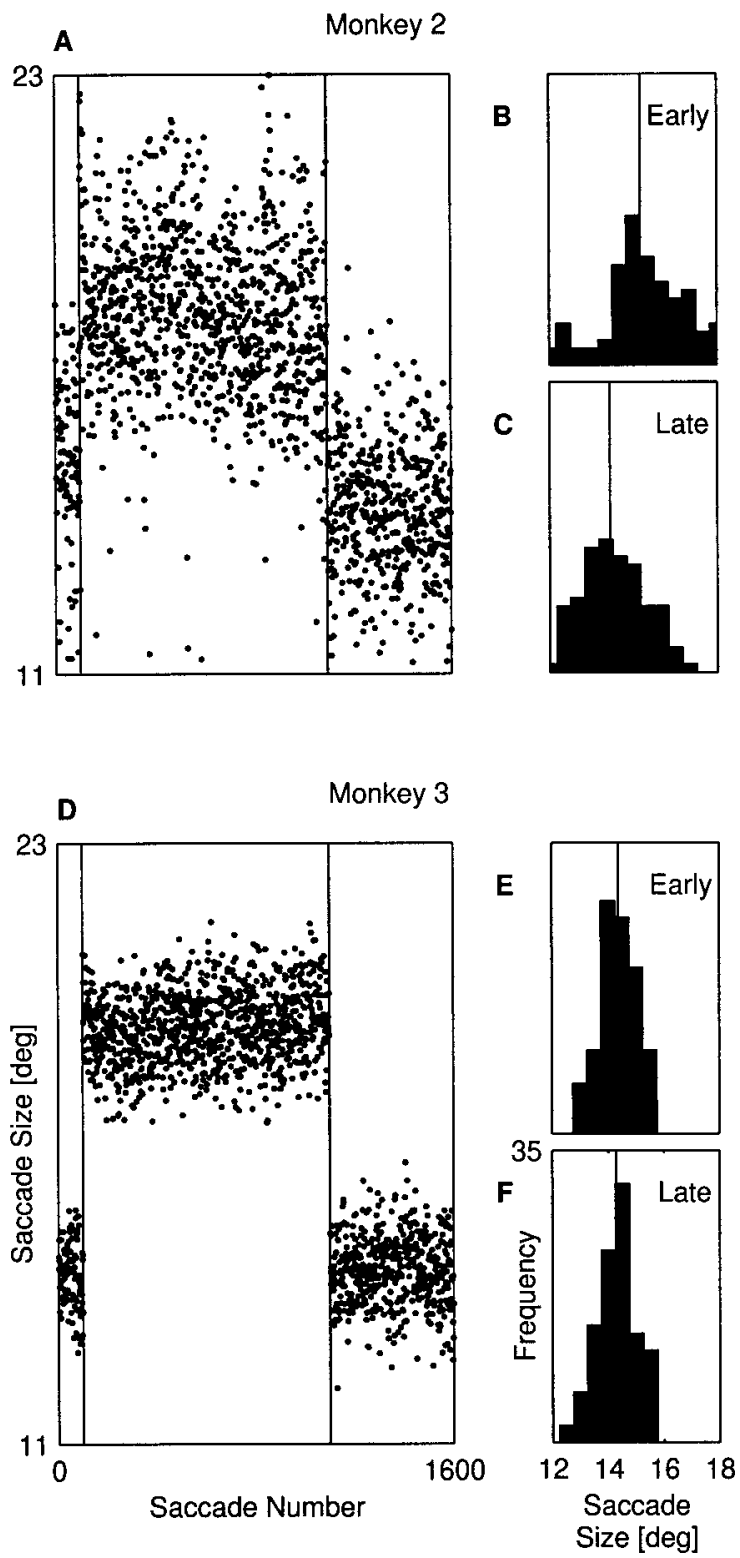

Figure 5. In the absence of saccadic adaptation, prolonged effort induces saccadic errors. A, Record of saccade size in the format of Figure 4; however, instead of adaptation trials, the monkey makes 200 control saccades. Saccades become smaller during the block. $B$, Histogram of first 100 trials; $C$, histogram of last 100 trials (1501-1600). $D-F$, Same format for monkey 3 who has an intact cerebellum. There is no analogous decrease in saccade size.

paradigm but is related to the loss of a cerebellar mechanism for continuous recalibration of saccades.

\section{Accuracy of saccades in other directions}

Saccade accuracy was most strongly affected in one of the four cardinal directions for each of the two monkeys; leftward for monkey 1 and rightward for monkey 2. In both animals, the direction opposite the one that was most severely affected was more mildly hypometric. The vertical dysmetrias for both animals are much smaller. Vertical hypermetria might reflect the presence of residual cortex deep in the sulci (see next section, Histological reconstruction). Table 1 presents the relevant measurements of saccade amplitude in each of the four directions. Recovery was virtually complete for the most severely affected directions. The more mild dysmetrias showed less long-term recovery. Because saccades in the present study were to targets at $15^{\circ}$ eccentricity, a dysmetria of $<10 \%$ represents an error, which would still leave the target within the fovea. The 20 and $32 \%$ dysmetrias represent 3 and $5^{\circ}$ errors, which may be more powerful stimuli for initiating a long-term recalibration of saccade amplitude.

\section{Histological reconstruction}

Figures 6 and 7 illustrate parasagittal sections through the center of the lesions (for review, see Madigan and Carpenter, 1971). The lesion in monkey 1 (Fig. 6) was primarily confined to the vermis and extends from lobule Vb to VIIIA. Laterally, the lesion slightly invaded the left hemisphere. The cerebellar nuclei, including fastigial and interpositus, were intact. The lesion in monkey 2 (Fig. 7) is more symmetric relative to the midsagittal plane and was entirely confined to the vermis. Lobule VIc in this monkey is in part intact. Caudally, the lesion extends slightly beyond the oculomotor vermis to include lobule VIIIA. Thus, the lesions cover most of the oculomotor vermis as determined by Noda (Fujikado and Noda, 1987; Noda and Fujikado, 1987a) with some slight invasion of bordering regions but sparing the deep cerebellar nuclei.

Saccadic direction is thought to be systematically mapped onto the surface of the saccadic area of the vermis (Ron and Robinson 1973; Noda and Fujikado 1987a,b). Because this map is organized so that the lateral dimension of the vermis corresponds to the horizontal and the anteroposterior to the vertical, vertical saccades are probably primarily represented by cortex deep within the sulci. Therefore, we conclude that the representation of horizontal saccades in the posterior vermis is nearly completely destroyed by the lesions. It seems likely that the little remaining cortex deep in the posterior vermis is probably associated primarily with vertical saccades. This corresponds to the limited dysmetria and the occurrence of some hypermetria in the vertical dimension.

\section{DISCUSSION}

\section{Summary of results}

We evaluated the effects of lesions that were restricted to the oculomotor posterior vermis as delineated by Noda (Fujikado and Noda, 1987; Noda and Fujikado, 1987a,b; Yamada and Noda, 1987; Sato and Noda, 1992; Ohtsuka and Noda, 1995). As expected, the lesions resulted in early postlesion saccadic hypometria in some directions. The main new findings reported here are as follows. (1) Contrary to expectations, there was recovery from hypometria over time. The recovery of the average saccade size was complete (in the directions in which the hypometria was severe in the early postlesion period). Individual saccades remained, however, less precise than before the lesion. (2) In the directions of severe initial dysmetria, the lesion abolished rapid saccadic adaptation. In contrast to the hypometria, which recovered, the abolition of adaptation was permanent. (3) In the absence of adaptation, repetition of the same saccade resulted in slow continuous reduction in saccade size.

\section{Speculation: two anatomically segregated processes of saccadic adaptation}

Several aspects of our findings may look paradoxical. For instance, the increased variability of saccade size might seem to be a secondary effect of the dysmetria, but it persists even after the recovery. The following interpretation, although speculative, is 
Table 1. Impact of lesions on saccades in different directions

\begin{tabular}{|c|c|c|c|c|c|c|c|c|c|c|}
\hline & & \multicolumn{3}{|c|}{ Saccadic gain } & \multicolumn{3}{|c|}{ Significance } & \multicolumn{3}{|l|}{$n$} \\
\hline & & Prelesion & Early post & Late post & $\begin{array}{l}\text { Pre versus } \\
\text { early }\end{array}$ & $\begin{array}{l}\text { Early versus } \\
\text { late }\end{array}$ & $\begin{array}{l}\text { Pre versus } \\
\text { late }\end{array}$ & Prelesion & Early post & Late post \\
\hline \multirow[t]{4}{*}{ Monkey 1} & Right & $100 \pm 4$ & $93 \pm 8$ & $92 \pm 11$ & $*$ & 0.30 & $*$ & 120 & 127 & 205 \\
\hline & Left & $97 \pm 3$ & $77 \pm 6$ & $98 \pm 7$ & * & $*$ & 0.24 & 120 & 129 & 206 \\
\hline & Up & $100 \pm 3$ & $103 \pm 5$ & $104 \pm 5$ & $*$ & 0.06 & $*$ & 122 & 135 & 212 \\
\hline & Down & $100 \pm 4$ & $91 \pm 5$ & $96 \pm 6$ & $*$ & $*$ & $*$ & 113 & 134 & 205 \\
\hline \multirow[t]{4}{*}{ Monkey 2} & Right & $100 \pm 4$ & $69 \pm 6$ & $100 \pm 10$ & $*$ & $*$ & 0.55 & 1114 & 151 & 177 \\
\hline & Left & $94 \pm 4$ & $88 \pm 8$ & $90 \pm 8$ & $*$ & $*$ & $*$ & 1117 & 140 & 170 \\
\hline & $\mathrm{Up}$ & $95 \pm 4$ & $94 \pm 5$ & $100 \pm 5$ & $*$ & $*$ & $*$ & 1137 & 138 & 178 \\
\hline & Down & $97 \pm 4$ & $98 \pm 5$ & $102 \pm 5$ & 0.19 & * & $*$ & 1143 & 141 & 177 \\
\hline
\end{tabular}

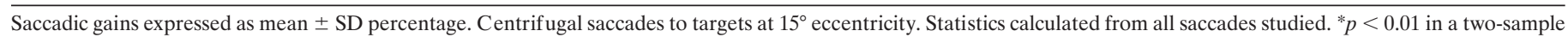
$t$ test.
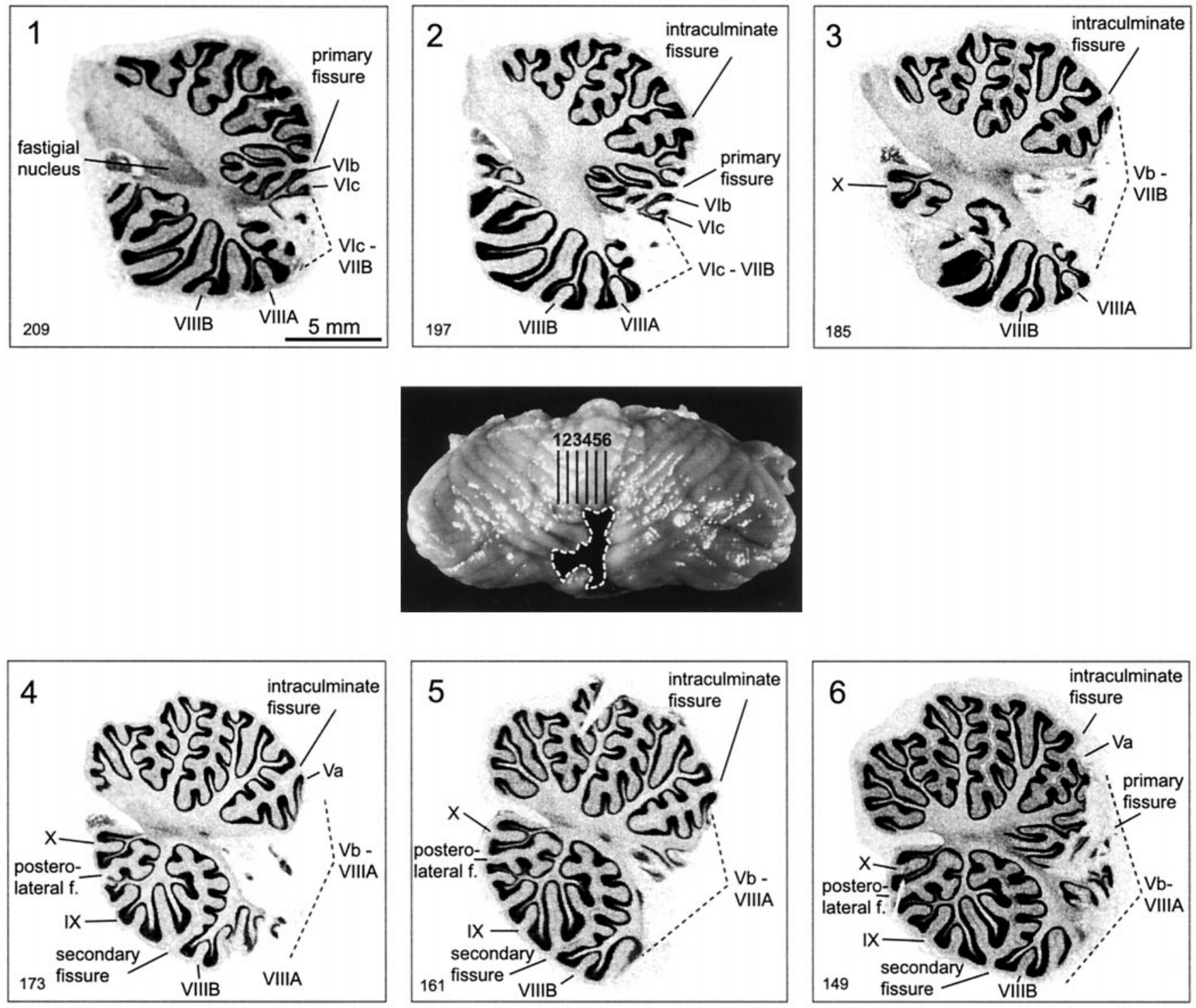

Figure 6. Histological sections through the lesion of monkey 1 . Sections are $60-\mu$ m-thick, Nissl stained. The calibration mark shown in section 1 is valid for all sections. Section numbers in the block are specified. Every 12th section is shown; that is, the distance between displayed sections is $0.72 \mathrm{~mm}$. 

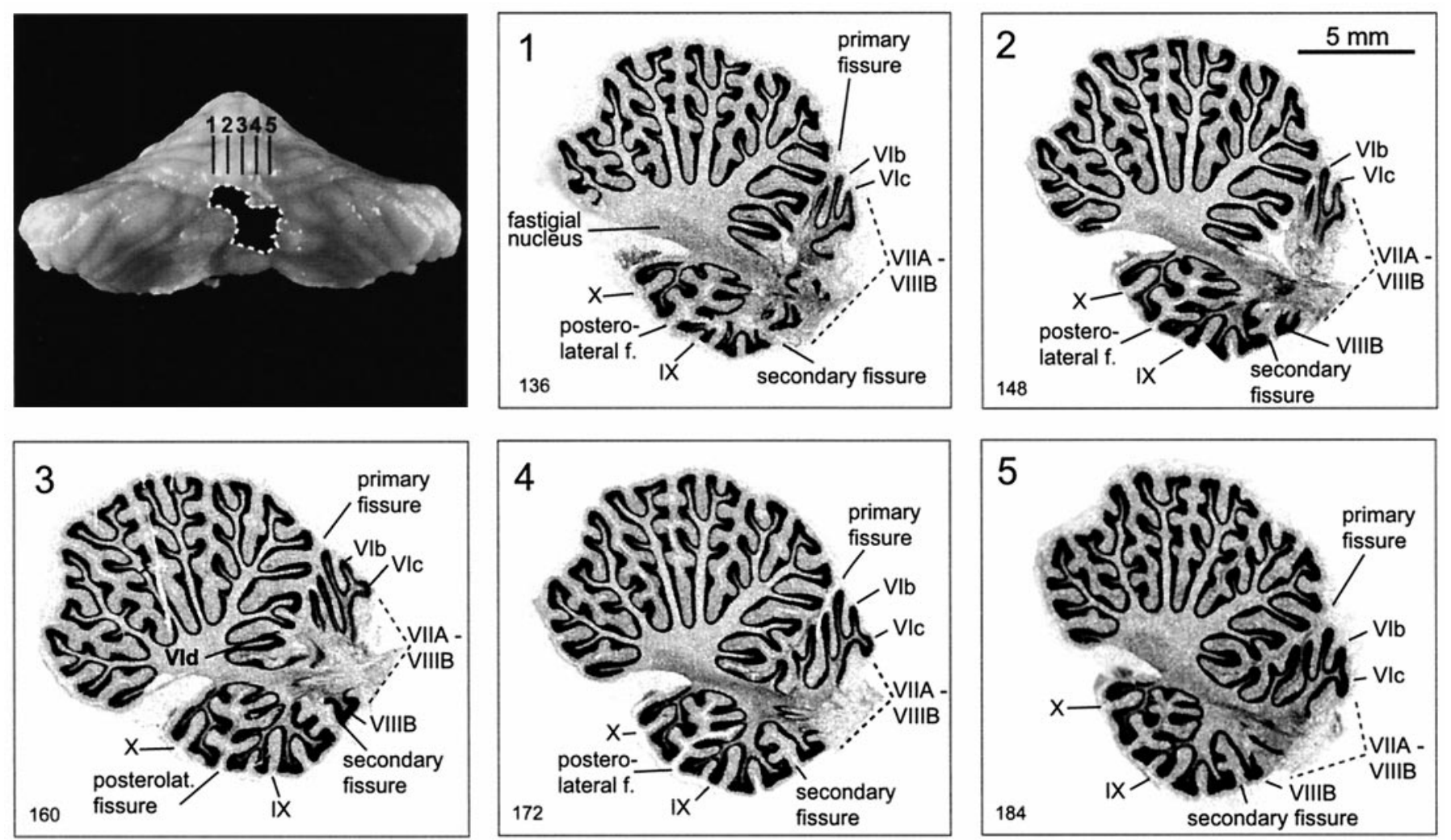

Figure 7. Histological sections through the lesion of monkey 2. Sections are $60-\mu \mathrm{m}$-thick, Nissl stained. The scale bar shown in section 2 is valid for all sections. Section numbers in the block are specified. Every 12th section is shown; that is, the distance between displayed sections is $0.72 \mathrm{~mm}$.

consistent with all aspects of our data and also with previous studies in which the fastigial nucleus was ablated in addition to the vermis (Optican and Robinson, 1980; see introductory remarks).

We propose that two processes adjust for the dysmetria. The two processes differ by the rate with which they adjust the saccadic gain (their "time constant"). A rapid process critically depends on functional posterior vermis cortex. A slow process is revealed in the absence of this region of cerebellar cortex; it might depend on the fastigial nuclei. In the intact cerebellum, the distinction between the two processes is obscured because both act in synergy to maintain saccadic accuracy with the rapid, cerebellar cortical process dominating the slower, noncortical.

Below, we shall examine how this proposition might relate to our data.

\section{Hypometria is consistent with previous data}

Our finding of hypometria, not hypermetria, is primarily consistent with previous studies. Buettner and Fuhry (1995) recently summarized the issue of saccadic hypometria versus hypermetria. Fastigial nuclei lesions result in hypermetria. Bilateral cortical lesions result in hypometric or normometric saccades. Only partial cortical lesions, primarily unilateral lesions, may result in hypermetria. Confounding factors here are the imperfect shape of surgical lesions, interindividual differences in the precise location of the oculomotor vermis, and the imprecise relationship of the functional to the anatomical midline (Noda and Fujikado, 1987a). Nevertheless, Buettner and Fuhry's conclusions are clearly supported by both our data and the data of Takagi et al. (1998).

\section{Increase of variability}

Takagi et al. (1998) found that posterior vermis lesions significantly increase saccade size variability. Figures 3 and 4 corroborate Takagi et al.'s report. They further show that the increased variability persists despite the complete recovery of the mean saccade size.

The increase in variability can be explained as failure of rapid but not slow adaptation. Slow adaptation acts as a high-pass filter. It corrects for slow gain errors and keeps the mean gain accurate (for sufficiently large samples). However, it fails to cope with high-frequency gain errors. These rapid errors of the gain present themselves as increased variability of saccade size.

Increase in saccade size variability was also observed after transient inactivation of the fastigial nuclei (Robinson et al., 1993). Robinson (1995) suggested that one function of the cerebellum is to reduce the large variability typical of neuronal activity to the remarkably low variability of movement size. Robinson's speculation is relevant to specifically rapid adaptation.

\section{Is the recovery mediated by adaptation?}

In the introductory remarks, we distinguished between traditional slow adaptation, induced by extraocular muscle paresis (Kommerell et al., 1976; Optican and Robinson, 1980), and rapid adaptation (McLaughlin, 1967; Fitzgibbon et al., 1986; Straube et al., 1997). Scudder et al. (1998) suggest that the two adaptation procedures reflect the same mechanism. Does Scudder's conclusion fit our results? Scudder studied monkeys with intact cerebella. Because in the intact cerebellum both processes work in synergy, if our two-process speculation actually holds, the rapid process would predominantly influence the rate of adaptation. 
Thus, Scudder's conclusion of a single process of saccadic adaptation does not extend to the lesioned monkeys. On the other hand, if Scudder's conclusion is valid, then the slow process revealed by the lesion is different from the one that follows extraocular muscle paresis.

Is the recovery from dysmetria mediated by any form of sensorimotor adaptation at all? The key feature of visuomotor adaptation is that it is invoked by vision; it is a process in which the precision of saccades is modified according to their visual outcome. If the gain modification was not based on vision, the precision of the recovery we observed would be remarkable. It is obvious that the large initial saccadic hypometria results in a significant loss of vision. During recovery, the monkey's vision is not restricted in any way. Therefore, the most likely possibility is that some form of sensorimotor adaptation mediates recovery.

\section{Relationship to neurological studies}

The accepted neurological sign of posterior vermis lesions is saccadic dysmetria (Leigh and Zee, 1990; Botzel et al., 1993), although it is not always present (Botzel et al., 1993). There is large variation among patients in the extent of damage to the cerebellar cortex, deep nuclei, or both. The times after the lesion at which the observations are made are highly variable (as after stroke) or undefined (as in patients with degenerative diseases). Thus, the differential effects of nuclear and cortical lesions cannot be addressed adequately in the clinical literature.

\section{Does the continuous reduction of saccade amplitude represent fatigue?}

The role of rapid saccadic adaptation has been a matter of speculation since the procedure for inducing it was invented (McLaughlin, 1967). McLaughlin was the first to suggest that this may reflect a mechanism for overcoming fatigue. Our data are consistent with this view. It seems reasonable that repetition of the same movement hundreds of times in a row would result in muscle fatigue, although additional evidence would be needed to prove the point.

We suggest that the oculomotor vermis is continuously active in adjusting and calibrating the oculomotor system, a process that is reflected in rapid saccadic adaptation. This mechanism is specifically involved in preventing the continuous reduction caused by fatigue. Thus, rapid saccadic adaptation would be a continuously active process, highly valuable for our ability to continue to use visual perception at the end of the day.

\section{Relationship to previous studies}

Although the posterior vermis and the fastigial nucleus are known to be involved in saccadic adaptation, the conclusion is based on a small number of monkeys. Moreover, in all but one study (Takagi et al., 1998), the distinction between cerebellar cortex and deep nuclei did not receive adequate attention. The very recent study of Takagi et al. (1998) is a welcome exception. These investigators presented important evidence implicating the posterior vermis in the control of saccade accuracy, dynamics, and latency. Our results differ in three points: (1) Takagi et al. observed only limited recovery from dysmetria, (2) they reported some recovery of adaptation, and (3) they did not observe or report on the "fatigue" effect, the gradual decrement in saccade amplitude over a long series of trials, that we describe.

These differences probably stem from various sources. The variability in saccade size may make the extent of adaptation harder to assess when studying smaller saccades and decreasing magnification as in Takagi et al. (1998). Another possibility for the discrepancy in results may relate to the extent to which the vermian lesions were bilateral and symmetrical. Takagi et al. (1998) reported large differences among the three monkeys that they studied. The deficits that they observed in their Monkey 1 were significantly different from those seen in their Monkeys 2 and 3. They suggested that the observed differences might relate to the anatomy of the lesions, which were symmetric in Monkey 1 , but asymmetric in Monkeys 2 and 3. The behavioral data that they reported for their Monkey 1 is similar in many respects to that of our monkeys. Their Monkey 1 failed to adapt after the lesion, whereas their Monkeys 2 and 3 recovered adaptation (their Fig. 11).

Although there are important points of agreement, the data from the two studies cannot simply be pooled. The lesions in our two monkeys were bilateral but not perfectly symmetrical in our monkey 1 . The exact damage to each side of this functional region is not established, because the functional midline may not be coextensive with the anatomical midline. Despite these reservations, the data from the two studies makes it clear that the posterior vermis regulates the amplitude of saccadic eye movements and is probably essential for the short-term adaptation.

\section{REFERENCES}

Albano JE, King WM (1989) Rapid adaptation of saccadic amplitude in humans and monkeys. Invest Ophthalmol Vis Sci 30:1883-1893.

Aschoff JC, Cohen B (1971) Changes in saccadic eye movements produced by cerebellar cortical lesions. Exp Neurol 32:123-133.

Barash S, Melikyan A, Sivakov A, Tauber M (1998) Shift of visual fixation dependent on background illumination. J Neurophysiol 79:2766-2781.

Botzel K, Rottach K, Buettner U (1993) Normal and pathological saccadic dysmetria. Brain 116:337-353.

Buettner U, Fuhry L (1995) Eye movements. Curr Opin Neurol 8:77-82.

Fitzgibbon EJ, Goldberg ME, Segraves MA (1986) Short term saccadic adaptation in the monkey. In: Adaptive processes in visual and oculomotor systems (Keller BL, Zee DS, eds), pp 329-333. Oxford: Pergamon.

Fujikado T, Noda H (1987) Saccadic eye movements evoked by microstimulation of lobule VII of the cerebellar vermis of macaque monkeys. J Physiol (Lond) 394:573-594.

Glickstein M (1992) The cerebellum and motor learning. Curr Opin Neurobiol 2:802-806.

Goldberg ME, Musil SY, Fitzgibbon EJ, Smith M, Olson CR (1993) The role of the cerebellum in the control of saccadic eye movements. In: Role of the cerebellum and basal ganglia in voluntary movement (Mano N, Hamada I, DeLong MR, eds), pp 203-211. Amsterdam: Elsevier Science.

Ito M (1998) Cerebellar learning in the vestibulo-ocular reflex. Trends Cognit Sci 2:313-321.

Judge SJ, Richmond BJ, Chu FC (1980) Implantation of magnetic search coils for measurement of eye position: an improved method. Vision Res 20:535-538.

Kommerell G, Olivier D, Theopold H (1976) Adaptive programming of phasic and tonic components in saccadic eye movements. Investigations of patients with abducens palsy. Invest Ophthalmol 15:657-660.

Leigh RJ, Zee DS (1990) The neurology of eye movements. Philadelphia: Davis.

Madigan Jr JC, Carpenter MB (1971) Cerebellum of the rhesus monkey. Baltimore: University Park.

McLaughlin SC (1967) Parametric adjustment in saccadic eye movements. Percept Psychophys 2:359-362.

Noda H, Fujikado T (1987a) Topography of the oculomotor area of the cerebellar vermis in macaques as determined by microstimulation. J Neurophysiol 58:359-378.

Noda H, Fujikado T (1987b) Involvement of Purkinje cells in evoking saccadic eye movements by microstimulation of the posterior cerebellar vermis of monkeys. J Neurophysiol 57:1247-1261. 
Ohtsuka K, Noda H (1995) Discharge properties of Purkinje cells in the oculomotor vermis during visually guided saccades in the macaque monkey. J Neurophysiol 74:1828-1840.

Optican LM, Robinson DA (1980) Cerebellar-dependent adaptive control of primate saccadic system. J Neurophysiol 44:1058-1076.

Raymond JL, Lisberger SG, Mauk MD (1996) The cerebellum: a neuronal learning machine? Science 272:1126-1131.

Ritchie L (1976) Effects of cerebellar lesions on saccadic eye movements. J Neurophysiol 39:1246-1256.

Robinson FR (1995) Role of the cerebellum in movement control and adaptation. Curr Opin Neurobiol 5:755-762.

Robinson FR, Straube A, Fuchs AF (1993) Role of the caudal fastigial nucleus in saccade generation. II. Effects of muscimol inactivation. J Neurophysiol 70:1741-1758.

Ron S, Robinson DA (1973) Eye movements evoked by cerebellar stimulation in the alert monkey. J Neurophysiol 36:1004-1022.

Sato H, Noda H (1992) Saccadic dysmetria induced by transient func- tional decortication of the cerebellar vermis. Exp Brain Res [Erratum 89:690] 88:455-458.

Scudder CA, Batourina EY, Tunder GS (1998) Comparison of two methods of producing adaptation of saccade size and implications for the site of plasticity J Neurophysiol 79:704-715.

Straube A, Fuchs AF, Usher S, Robinson FR (1997) Characteristics of saccadic gain adaptation in rhesus macaques. J Neurophysiol 77:874-895.

Takagi M, Zee DS, Tamargo RJ (1998) Effects of lesions of the oculomotor vermis on eye movements in primate: saccades. J Neurophysiol 80:1911-1931.

Thompson RF, Bao S, Chen L, Cipriano BD, Grethe JS, Kim JJ, Thompson JK, Tracy JA, Weninger MS, Krupa DJ (1997) Associative learning. Int Rev Neurobiol 41:151-189.

Yamada J, Noda H (1987) Afferent and efferent connections of the oculomotor cerebellar vermis in the macaque monkey. J Comp Neurol 265:224-241. 